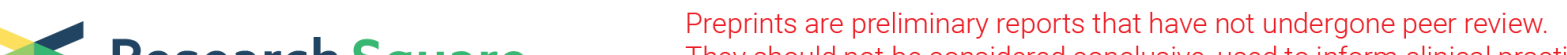 $\begin{array}{ll}\text { Research Square } & \begin{array}{l}\text { They should not be considered conclusive, used to inform clinical practice, } \\ \text { or referenced by the media as validated information. }\end{array}\end{array}$
}

\section{SLUG is Enhanced by Chemotherapeutics and Functions to Promote Invasion and Metastasis by Directly Targeting MMP3 in Cervical Cancer}

\section{Senwei Jiang}

Sun Yat-sen University Cancer Center

\section{Lan Zhang}

Yunnan Cancer Hospital

\section{Shuting Huang}

Guangdong General Hospital: Guangdong Provincial People's Hospital

\section{Yun Zhou}

Sun Yat-sen University Cancer Center

\section{Yanling Feng}

Sun Yat-sen University Cancer Center

\section{Linjing Yuan}

Sun Yat-sen University First Affiliated Hospital

\section{Yinan Jiang}

Sun Yat-sen University Cancer Center

\section{Xiaojing Zheng}

Sun Yat-sen University Cancer Center

Jing Xu

Sun Yat-sen University Cancer Center

\section{Rongzhen Luo}

Sun Yat-sen University Cancer Center

\section{Weihua Jia}

Sun Yat-sen University Cancer Center

Min Zheng ( $\nabla$ zhengmin@sysucc.org.cn)

Sun Yat-sen University Cancer Center

\section{Research}

Keywords: SLUG, MMP3, cervical cancer, neoadjuvant chemotherapy, metastasis

Posted Date: October 7th, 2020

DOl: https://doi.org/10.21203/rs.3.rs-86060/v1 
License: (c) (i) This work is licensed under a Creative Commons Attribution 4.0 International License. Read Full License 


\section{Abstract}

\section{Background}

Neoadjuvant chemotherapy(NACT) is a widely used strategy in the treatment of locally advanced cancers. NACT followed by radical surgery for patients in FIGO stages IB2-IIB has proven to reduce tumor volume and numbers. Under certain conditions, however, NACT followed by radical surgery has not been found to improve overall survival, but rather to accelerate cancer growth and metastasis in current studies.

\section{Methods}

In this study, 35 paired pre/post-NACT cervical cancer tissues and 167 cervical cancer samples were examined by immunohistochemical analysis to investigate the role of SLUG in invasion and metastasis. Functional assays and a CHIP assay were performed to determine SLUG's downstream genes and pathways. A lymph node metastatic mouse model was used to study the effects of SLUG-and MMP3silencing on the malignant behavior of the cervical cancer cells.

\section{Results}

SLUG expression was potentially increased in cervical cancer tissues after neoadjuvant chemotherapy, which in turn positively correlated with pelvic lymph node metastasis. The expression of SLUG following neoadjuvant chemotherapy was identified as a poor survival indicator. SLUG overexpression promoted metastasis in cervical cancer in both in vitro and in vivo models, whereas SLUG silencing had the opposite effect. Mechanically, SLUG is not a prerequisite for EMT activation in cervical cancer models, but contributes to the invasiveness and metastasis of cervical cancer cells by directly tethering to the recognition sequence GCCAGGTGC in the MMP3 promoter region.

\section{Conclusion}

Our results provide mechanistic insight into the potential pro-cancer effect of neoadjuvant chemotherapy, and demonstrates that the SLUG-MMP3 axis could serve as a target for improving the efficacy of chemotherapy.

\section{Background}

Cervical cancer is the most common gynecological malignancy of the genital tract, which in spite of advances in screening, diagnosis, prevention and treatment methods[1], continues to exhibit increased incidence and decreased average onset age. Formerly, the standard treatment for early stage cervical cancer consisted of radical surgery and radiotherapy, with administration of neoadjuvant chemotherapy (NACT) before surgery for patients in FIGO stages IB2-IIB, which has proven to reduce tumor volume and numbers[2]. However, NACT followed by radical surgery has not been found to improve overall survival, and may even be a contributing factor in increased recurrence[3]. Furthermore, recent studies have shown 
that chemotherapeutic drugs could alter the transcriptome of surviving cells and potentially promote cancer metastasis[4-6].

A previous study discovered using gene expression microarrays that the expression of SLUG in breast cancer cells is increased when the patient in question was treated with the chemotherapeutic drug cisplatin[7]. SLUG, a member of the Snail family of transcription factors, displays a unique conserved motif near the zinc fingers that is absent in other members[8]. A high expression of SLUG can be found in highly invasive cancer cells and tumor specimens, and is associated with cancer metastasis and poor survival $[9,10]$. As such, our intent was to explore whether SLUG expression in cervical cancer cells could also be increased by using NACT drugs. Although SLUG is a known epithelial-mesenchymal transition (EMT) inducing factor and clinical indicator across a variety of human cancers[11, 12], the function of SLUG in cervical cancer cells remains ambiguous.

In this study, we compared the expression of SLUG between pre-NACT and post-NACT cervical cancer tissues. Our analyses revealed an increased expression of SLUG in cancer cells following treatment via chemotherapeutic drugs. The SLUG expression was found to correlate with lymph node metastasis, and served as a prognostic marker. Overexpression of SLUG in cervical cancer cells led to increased invasive and metastasis capabilities both in vitro and in vivo. Mechanically, SLUG is not a requirement for EMT activation in cervical cancer models, but does contribute to the invasiveness and metastasis of cervical cancer cells by directly promoting MMP3 transcription. Our results indicate that SLUG is a cervical-cancerassociated gene, and could serve as a target for the development of new therapeutic strategies.

\section{Materials And Methods}

\subsection{Cervical cancer patients and cancer tissue samples}

The archived formalin-fixed paraffin-embedded tissue was taken from 202 diagnosed cervical cancer patients at Sun Yat-sen University Cancer Center, including 167 stage IB-IIB patients who underwent surgical resection between 2000 and 2008, with the addition of postoperative chemotherapy or radiotherapy if pathological risk factors were identified; 35 patients who received NACT (neoadjuvant chemotherapy) and surgical resection between 2007 and 2012 Stage IB-IIB patients. An inclusion criterion applied at patient enrollment was the absence of any anticancer therapy prior to treatment. The clinicopathological characteristics of the patients are summarized in supplementary Table S1.

All patients participated in outpatient follow-up and were monitored for recurrence at regular intervals of 3-6 months by history and physical examination, serum squamous cell carcinoma antigen level testing in squamous cell carcinoma patients, pelvic and abdominal ultrasound, and chest x-ray. If tumor recurrence or metastasis is suspected, further investigations, including CT and PET-CT scans, are performed. Biopsies are performed as necessary. Patients diagnosed with recurrence receive further treatment, including surgical resection, local ablative therapy, and chemotherapy with or without radiation therapy. 
The disease-free survival (DFS) was defined as the time interval from surgery to recurrence or, for patients with no survival data, the time interval from surgery to last observation (for censoring observations). The overall patient survival (OS) was defined as the interval from surgery to the patient's death or, for patients who survived, the interval from surgery to the last observation (for censored observations). Staging was performed according to the International Federation of Gynecology and Obstetrics (FIGO 2009) classification guidelines.

Two experienced pathologists graded scores and identified subtypes of the specimens according to World Health Organization criteria. All human specimens used in this study were approved by the Medical Ethics Committee of Sun Yat-sen University Cancer Center.

\subsection{Cells lines}

The human cervical cancer cell lines HeLa, SiHa, Caski and the human kidney cell line HEK-293T were obtained from the Cell Bank, Shanghai Institutes for Biological Sciences (Shanghai, China). Cells were grown in a DMEM or RPMI-1640 medium (Gibco, USA), supplemented with a 10\% fetal bovine serum (Gibco, USA).

\subsection{RNA extraction and quantitative real-time polymerase chain reaction}

Total RNA was extracted from cultured cells and fresh frozen cervical tissue using Trizol reagent (Life Technology) according to the manufacturer's protocols. Reverse transcriptase reactions were performed using MMLV Reverse Transcriptase Reagent (Promega) according to the manufacturer's instructions. Gene expression levels were normalized to the housekeeping genes $\beta$-actin and GAPDH. Reactions were performed in triplicate using a Roche LightCycler 480 II PCR system (Roche). Primer sequences are listed in Supplemental Table S2.

\subsection{Protein extraction and western blotting}

Protein extraction kits (KeyGen Biotech, China) were used to extract proteins.. Protein samples were treated with loading buffer (Thermo Fisher Scientific, USA) containing reducing agent for $10 \mathrm{~min}$ at $95^{\circ} \mathrm{C}$, resolved on $10 \%$ Tris- $\mathrm{HCl}$ polyacrylamide gels, and transferred to polyvinylidene fluoride membranes. After incubation of the primary antibody overnight $\left(4^{\circ} \mathrm{C}\right)$, HRP-conjugated antibodies and Immobilon Western Chemiluminescent HRP Substrate (Millipore Corporation, USA) were applied. A detailed list of the antibodies used can be found in Supplemental Table S3.

\subsection{Immunohistochemistry}

Immunohistochemistry (IHC) was performed in 2 steps according to the protocol (DakoCytomation, Denmark). After dewaxing, a hydration and endogenous peroxidase blocking procedure $\left(0.3 \% \mathrm{H}_{2} \mathrm{O}_{2}\right.$, $10 \mathrm{~min}$ ) was performed. Antigen retrieval was performed by boiling sections in $10 \mathrm{mM}$ citric acid buffer for 10 min. sections were incubated with primary antibody overnight at $4{ }^{\circ} \mathrm{C}$ and stained with cytokeratin isotype antibody as a negative control. The horseradish peroxidase-conjugated anti-mouse and anti- 
rabbit Dako EnVision system (DakoCytomation) was used as a secondary detection reagent, developed with 3,3'-diaminobenzidine (DAB). The expression of proteins in tissues was measured using the $\mathrm{H}$-score method. The final score was obtained by calculating the percentage of positively stained cells $(0 \%-100 \%)$ in each staining intensity category $(0-3)$. ( $3 *$ percentage of strong staining $)+(2 *$ percentage of moderate staining $)+(1 *$ percentage of weak staining), resulting in an $\mathrm{H}$-score ranging from $0-300$. IHC sections were examined by two independent pathologists, blinded to the clinical information of each patient. If the results of the two examinations were inconsistent, further evaluation was performed by a third pathologist.

\subsection{RNA interference}

Short interfering RNAs designated as specific for target genes, and their corresponding scrambled siRNA were purchased from IGE BIO (China); sequences are summarized in Supplementary Table S4. Transfection using Opti-mem medium (Life Technology) and Lipofectamine 3000 reagent (Life Technology) according to the manufacturer's protocols. After incubated for 6 hours at $37^{\circ} \mathrm{C}$, the medium was replaced with normal growth medium containing $10 \%$ fetal bovine serum. After 48 hours, the cells were harvested and the effect of gene silencing was tested by western blot.

\subsection{Plasmid constructs and lentiviral infection}

The overexpression plasmids, shRNA plasmids, and control plasmids were purchased from GeneCopoeia (Fullen Genetics, China). The Lenti-PacTM HIV expression packaging kit (GeneCopoeia) was used to transfect the 293FT packaging cell line according to the manufacturer's instructions. Virus-containing supernatants from 293FT cells were collected and then filtered through a $0.45 \mu \mathrm{m}$ filter. The filtered supernatant was added to $60 \%$ confluent cells and in the presence of $6 \mu \mathrm{g} / \mathrm{ml}$ polypropylene (Sigma, USA).After 2 days, the medium was replaced with complete growth medium containing $2 \mathrm{ug} / \mathrm{ml}$ puromycin to stabilize the transduced cells.

\subsection{Migration and invasion assays}

For the migration assay, $2 * 10^{5}$ tumor cells were placed in the chamber of each insert (BD, USA). For the invasion assay, $2^{\star} 10^{5}$ tumor cells were placed in the chamber coated with a 1:3 dilution of Matrigel (BD, USA): DMEM(Gibco, USA). Complete medium containing 10\% FBS was added to the lower chamber as a chemoattractant. After $8 \mathrm{~h}$ (for migration assay) and $48 \mathrm{~h}$ (for invasion assay) in the incubator, gently remove the upside cells from the chamber. The lower side migrating cells were stained with crystal violet and counted. Three independent experiments were performed.

\subsection{Chromation immunoprecipitation assay}

Tumor cells were treated with $1 \%$ formaldehyde to obtain protein cross-linked DNAs. Chromation immunoprecipitation assay (ChIP) assay was performed by using the SimpleChIP Enzymatic Chromatin IP Kit (Cell Signaling Technology, USA) following the manufacturer's protocol. Then, ChIP-enriched DNA was analyzed by qRT-PCR using Roche LightCycler 480 II PCR system. The antibodies used are listed in Supplementary Table S3. The primers for ChIP PCR are shown in Supplementary Table S5. 


\subsection{Animal experiments}

Animal experiments were conducted according to the experimental animal welfare guidelines of Sun Yatsen University. 5 weeks female BALB/c nude mice were purchased from the Guangdong Experimental Animal Center. Randomly divided the mice into four groups ( $n=9 /$ group), $2 * 10^{5}$ cells ( $\mathrm{SiHa}$-Vector/SiHaSLUG;SiHa-Vector-shMMP3/SiHa-SLUG-shMMP3) were injected into the foot pads as previously described[13]. 8 weeks later, the formed tumors and lymph nodes were removed and measured. Lymph nodes were estimated using volume $V=3 / 4^{\star}(d / 2)^{3}$. Sections were immunostained with anti-cytokeratin antibodies and analyzed.

\subsection{Statistical analysis}

The statistical analysis was performed using SPSS (version 16.0, SPSS Inc) and GraphPad Prism (version 6, GraphPad Software). All of the data are presented as the mean \pm standard errors of mean (SEM)except as otherwise specified. A Student's t test was used to calculate the $p$ values except as

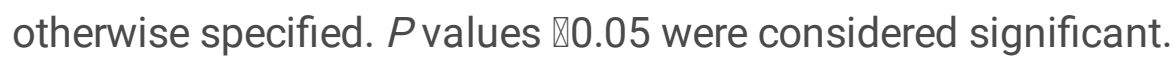

\section{Results}

\subsection{Chemotherapy enhances SLUG expression in cervical cancer}

To investigate whether SLUG expression is affected by NACT in the context of cervical cancer, 35 paired pre- and post-NACT tissues were examined using IHC, with SLUG being detected mainly in the nucleus. 12 patients had no obvious tumor residuals after NACT, and as such were excluded. Among the remaining 23 patients, in comparison with pre-NACT staining, post-NACT SLUG scores were increased in 14/23 (60.87\%) patients, remained stable in $3 / 23(13.04 \%)$ patients, and decreased in 6/23 $(26.09 \%)$ patients (Fig. 1A). To confirm the effects of chemotherapy drugs on cervical cancer, SiHa cells were treated with the chemotherapy drug cisplatin (0-64 $\mu \mathrm{M})$, a frontline NACT agent in cervical cancer, for $4 \mathrm{~h}$ and $24 \mathrm{~h}$ respectively. As a result, a dose-dependent increase of SLUG in both mRNA and protein levels (Fig. 1B) was observed. Collectively, these data demonstrate that the expression of SLUG in cervical cancer cells could be upregulated by chemotherapy.

\subsection{SLUG expression is correlated with lymph node metastasis, and is a prognostic marker in cervical cancer}

In order to discern the clinical significance of SLUG, an immunohistochemical analysis was performed on 169 cervical cancer samples. We found that SLUG was moderately expressed in most adjacent normal epithelial tissues, but displayed varying degrees of staining in tumor tissues. Based on the immunostaining score system, tumor samples were separated into a "high-expression" group (score $\geq 2, n$ $=125$ ) and a "low expression" group (score $22, n=44)(F i g .1 C)$. As shown in Table 1, SLUG expression did 
not show correlation with age, FIGO stage, differentiation, tumor size, lymphovascular space invasion, cervical invasion or uterine corpus invasion, but was correlated with lymph node metastasis $(P=0.002)$, distant metastasis and recurrence $(P=0.028)$. The association between SLUG expression in cervical cancer and survival time of patients was analyzed using the Kaplan-Meier method. The 10-year diseasefree survival (DFS) and 10-year overall survival (OS) time of the high SLUG expression group was shorter than that of low SLUG expression group $(P=0.020 ; P=0.135$, Fig. 1D). These clinical data suggest that SLUG could affect cervical cancer metastasis and serve as a prognostic marker for cervical cancer.

\subsection{SLUG enhances the invasive capabilities of cervical cancer cells}

Based on the above results, we hypothesized that SLUG may affect the motility of cervical cancer cells; as such, we decided to conduct further wound healing and Matrigel invasion assays. Using a lentiviral infection system, we maintained stable overexpression of SLUG in SiHa cells and silenced SLUG in Caski and HeLa cells. The levels of SLUG in these resultant cell lines were then determined by western blot analysis and qRT-PCR (Fig. 2A). Wound healing assays indicated that the cell migratory speed was not changed by SLUG overexpression or knockdown (Fig. 2B); transwell Matrigel invasion assays showed, however, that SLUG-overexpressing cells exhibited a higher invasive capacity than vector control cells, whereas SLUG-inhibited cells had a lower invasive capacity (Fig. 2C). These results suggest that SLUG promotes invasiveness in cervical cancer cells.

We then investigated the in vivo effects of SLUG on cervical cancer metastasis using a lymph node metastasis model. Metastatic activity was expected to occur primarily in the popliteal and inguinal lymph nodes (Fig. 3A). Equal amounts of log-phase SLUG overexpression or control SiHa cells were injected into the footpads of nude mice, and injection-site associated tumors, popliteal and inguinal lymph nodes were collected after 8 weeks(Fig. 3B). As shown in Fig. 3C, no obvious difference is present in the volume of inguinal lymph nodes that formed as a result of SLUG overexpression cell and vector cell injection. However, the volumes of footpad tumors and popliteal lymph nodes that formed from SLUG overexpressing cells were significantly larger than those that formed from vector-control cells (Fig. $3 \mathrm{C}$ ). A remarkable increase of cytokeratin-positive tumor cells could be detected in the lymph nodes of the SLUG overexpressing group compared with the control-vector group (Fig. 3D). Notably, the ratios of metastatic to dissected and lymph nodes were found to be markedly higher in the SiHa-SLUG (100\% (7/7) for popliteal, $42.9 \%$ (3/7) for inguinal, 71.4\% (10/14) for all) group in comparison with the SiHa-vector group (57.1\% (4/7) for popliteal, 28.6\% (2/7) for inguinal, 35.7\% (6/14) for all) (Fig. 3E). Taken together, these results indicate that SLUG promotes lymph node metastasis in cervical cancer in vivo.

\subsection{SLUG does not play a role in EMT regulation in cervical cancer cells}

EMT is a process by which cancer cells lose their epithelial properties to acquire a mesenchymal phenotype and become motile and invasive, which is strongly associated with metastasis[14]. It has been reported that SLUG serves a crucial role in controlling EMT. In our study, we examined the morphological 
characteristics and EMT markers of the cell lines during exponential growth, and found no morphological changes in SLUG overexpression SiHa cells or SLUG silencing Caski cells(Fig. 4A). Western blot assays showed that both overexpression and knockdown of SLUG failed to alter the mesenchymal marker Vimentin of the epithelial marker E-cadherin (Fig. 4B), suggesting that SLUG levels are not critical for EMT activation in these tested cells' system. As SLUG-mediated invasion in cervical cancer cells is evidenced to be independent of EMT, exploration of other pathways that may contribute to the invasive and metastasis capabilities of cervical cancer cells is warranted.

\subsection{SLUG-stimulated MMP3 expression in cervical cancer cells}

As a commonly known transcription factor, SLUG fulfills its function by activating the transcription of downstream target genes. In our study, we used an Aligent SurePrint G3 Human GE $8 \times 60 \mathrm{~K}$ Microarray to compare whole transcriptome gene expressions between SiHa-SLUG and SiHa-lv105 cells during exponential growth. The heat map in Fig. $4 \mathrm{C}$ shows the 55 genes that exhibited the most drastic changes, of which MMP3, one of the most widely expressed matrix metalloproteinases that can degrade type II, III, IV, IX and X collagens, was found to be enhanced more than 5 times in SLUG overexpressing cells.

Previous studies have demonstrated that MMP3 is an important mechanism involved in tumor invasion and metastasis[15]; this prompted us to investigate whether it is MMP3 that contributes to SLUGassociated invasiveness and metastasis. As shown in Fig. 5A-B, MMP3 expression was increased in SLUG overexpressing cells but decreased in SLUG knockdown cervical cancer cells at the mRNA and protein levels in comparison with control cells, suggesting that SLUG transcriptionally upregulates MMP3 in cervical cancer cells. A bioinformation analysis(JASPAR database) showed that there are possible SLUG binding elements located between -769 to $-761,-1021$ to $-1013,-1355$ to -1347 and -1552 to -1544 bp on the MMP3 promoter (Fig. 5C). A chromatin immunoprecipitation (ChIP) analysis was deployed to examine SLUG-binding sites within the MMP3 promoter region; results indicated that SLUG selectively bound to a region (-1552 to $-1544 \mathrm{bp}$ ) of the MMP3 promoter, but not to the sites located at other positions (Fig. 5E), which implies that SLUG upregulates MMP3 expression by targeting the MMP3 promoter. We also use ChIP analysis examine SLUG-binding sites within the MMP1 promoter region, results indicated that SLUG not bound to the MMP1 promoter (Fig. 5D, F).

\subsection{MMP3 is required for SLUG-induced invasion and metastasis}

We further confirmed that MMP3 is a requirement for SLUG-induced invasion and metastasis. As shown in Fig. 5G, silencing MMP3 reduced the ability of SLUG overexpressing cells to induce invasion. In addition, we found that downregulation of MMP3 reduced the volume and number of cytokeratin-positive tumor cells in the lymph nodes of transfected mice (Fig. 6A-C); the ratios of metastatic to total dissected lymph nodes were markedly reduced from $94.4 \%$ to $55.6 \%$ (Siha-SLUG) and 61.1-50.0\% (Siha-Vector) (Fig. 3E,6D). Taken together, these results demonstrate that MMP3 is a key mediator in SLUG-induced invasion and metastasis. 


\section{Discussion}

With the intent to gain new insight into the mechanism by which NACT influences cancer invasion and metastasis, we performed this study and discovered that chemotherapeutics enhanced SLUG expression level both in cervical cancer tissues and cell lines. Our results show that SLUG expression is associated with lymph node metastasis and can serve as an adverse prognostic factor. Similar to the results in lung, gallbladder and breast cancer[16-18],SLUG overexpression was established to promote cervical cancer invasion and metastasis. Furthermore, our experiments demonstrate that SLUG-mediated invasion and metastasis are not EMT dependent, but occur via direct targeting of MMP3 transcription.

NACT is widely used in the treatment of locally advanced cervical cancer in many developing countries, including China. However, NACT followed by radical surgery has not been found conducive to improved overall survival, and has even been considered as potentially contributive to recurrence[19,20]. Studies have proven chemotherapy to be a double-edged sword: its cytotoxicity translates to effective cancer treatment, but can also be procancer by inducing changes in cancer and/or host cells[21]. Nonetheless, the precise mechanisms by which chemotherapy-induced procancer processes occur remain poorly understood; in breast cancer, paclitaxel therapy for patients with TLR4-expressing tumors has been known to possibly activate systemic inflammatory circuits that promote angiogenesis, lymphangiogenesis, and metastasis[22], while chemotherapy has been reported to promote selection of chemoresistant and disseminating tumor cells endowed with properties of cancer stem cells (CSCs) through activation of autocrine and paracrine self-renewing/survival pathways, promoted jointly by therapy-selected tumor and stromal cells[23]. In our study, SLUG was found to be potentially enhanced in cervical cancer tissues after neoadjuvant chemotherapy, which promotes cancer cells invasion and metastasis by directly targeting MMP3.

Members of the Snail family function as transcription factors and are involved in a variety of cellular biological processes across various types of cancers, with functions including but not limited to cell transcriptional regulation, formation of repressive chromatin structure, cellular signaling, and developmental processes[24]. The role of the Snail family in different types of tumor cells may be different. Snail elicits collective migration in squamous cell carcinoma by inducing the expression of claudin-11, and the Snail-claudin-11 axis prompts the formation of circulating tumour cell clusters[25]. Snail also induces transactivation of target genes (TNF-a, CCL2 and CCL5) to remodel tumor microenvironments[26]. In breast cancer cells, Slug weakens the invasion ability of breast cancer cells and reverses the Jagged1-induced EMT process with significantly decreased expression of vimentin and increased expression of E-cadherin[18]. In our study, we examined the morphological characteristics and EMT markers of cervical cancer cell lines, and our results suggest that SLUG expressions are not critical for EMT activation of cervical cancer cell lines; SLUG-mediated cervical cancer cell invasion is independent of EMT.

The matrix metalloproteinase family (MMPs) is a zinc-dependent endopeptidase that contains more than 20 members[27], and serve major functions in degrading extracellular matrix proteins and promoting 
tumors invasion and metastasis[28]. In addition, MMPs can regulate the biological processes of tumors by regulating the expression of other proteins[29]. MMP3 can degrade collagen types II, III, IV, IX and X, proteoglycans, fibronectin, laminin and elastin[30]. MMP3 can also activate other MMPs, such as MMP1, MMP7 and MMP9, thereby granting it a key role in connective tissue remodeling. The enzyme is also considered to be involved in wound repair, atherosclerosis progression and tumorigenesis[31]. In another study, the expressions of SLUG, MMP1, MMP3, and MYC were found to be correlated, suggesting that SLUG may play a role as a transcription factor for MMP1 and MMP3[32]. Our gene microarray results showed that MMP1 and MMP3 were significantly upregulated in SLUG overexpressing cells, which was further confirmed by quantitative PCR and western blot. SLUG was found to directly bind to the upstream sequence of the promoter to promote MMP3 transcription.

In summary: Cisplatin can induce the expression of SLUG in cervical cancer cells. SLUG is a metastasispromoting gene, which has significant value in predicting postoperative recurrence for cervical cancer patients. SLUG promotes MMP3 transcription by directly binding to the promoter position of MMP3, which in turn facilitates cervical cancer cell invasion and metastasis. Exploring SLUG-regulated genes will reveal further clues as to its biological roles and contribute to the more general understanding of the Snail family and the mechanisms underlying the metastasis of cervical cancer.

\section{Conclusions}

In this study, we establish that the expression of SLUG in cervical cancer cells can be upregulated by chemotherapy, which correlates with cancer metastasis and poor survival. In addition, our experiments demonstrate that SLUG-mediated invasion and metastasis are not EMT-dependent, but occur via direct targeting of MMP3 transcription. Collectively, our results offer mechanistic insight into the potential procancer effects of neoadjuvant chemotherapy, and demonstrate that the SLUG-MMP3 axis could serve as a target for improving the efficacy of chemotherapeutic treatments.

\section{Abbreviations}

SLUG, SNAI2

Snail Family Transcriptional Repressor 2;

MMP3

Matrix Metallopeptidase 3;

MMP1

Matrix Metallopeptidase 1;

NACT

neoadjuvant chemotherapy;

PET-CT

Positron emission tomography-computed tomography;

CHIP

Chromation immunoprecipitation; 
PLNM

pelvic lymph node metastasis;

EMT

epithelial-mesenchymal transition;

DFS

disease free survival;

OS

Overall survival;

FIGO

international federation of gynecology and obstetrics.

\section{Declarations}

\section{Ethics approval and consent to participate}

All procedures involving human participants were performed in accordance with Sun Yat-sen University Cancer Center ethical committee and with the 1964 Declaration of Helsinki and its later amendments or comparable ethical standards. All patients provided their written informed consent. The study protocol was approved by the Sun Yat-sen University Cancer Center Committee on human research.

\section{Consent for publication}

Written informed consent for publication was obtained from all the participants.

\section{Availability of data and materials}

The datasets used and analyzed during the current study are available from the corresponding author on reasonable request.

\section{Competing interests}

The authors declare that they have no competing interests.

\section{Funding}

This work was supported by grants from the National Natural Science Foundation of China (Grant numbers 81672863, 81702550, 81872434, 81802614), Guangdong Basic and Applied Basic Research Foundation (Grant number 2019A1515011191) , and the Project of State Key Laboratory of Oncology in South China (Grant number 030041060004).

\section{Authors' contributions}

SJ, LZ and MZ conceptualization; SJ, LZ and YZ. data curation; SJ, LZ, YZ, YF, LY, YJ, and XZ data collection and analysis; SJ, LZ, YZ, JX, RL and WJ methodology; SJ and LZ wrote and edited this 
manuscript; $\mathrm{SH}$ and $\mathrm{MZ}$ read and revised this manuscript; $\mathrm{MZ}, \mathrm{LZ}, \mathrm{SH}$ and $\mathrm{LY}$ funding acquisition; $\mathrm{MZ}$ project administration. All authors read and approved the final manuscript.

\section{Acknowledgements}

Not applicable.

\section{References}

1. Bray F, Ferlay J, Soerjomataram I, Siegel RL, Torre LA, Jemal A. Global cancer statistics 2018 : GLOBOCAN estimates of incidence and mortality worldwide for 36 cancers in 185 countries. Cancer J Clin. 2018;68:394-424.

2. Cohen PA, Jhingran A, Oaknin A, Denny L. Cervical cancer. Lancet. 2019;393:169-82.

3. Gupta S, Maheshwari A, Parab P, Mahantshetty U, Hawaldar R, Sastri Chopra S, et al. Neoadjuvant Chemotherapy Followed by Radical Surgery Versus Concomitant Chemotherapy and Radiotherapy in Patients With Stage IB2, IIA, or IIB Squamous Cervical Cancer: A Randomized Controlled Trial. Journal of clinical oncology: official journal of the American Society of Clinical Oncology. 2018;36:1548-55.

4. Shaffer SM, Dunagin MC, Torborg SR, Torre EA, Emert B, Krepler C, et al. Rare cell variability and druginduced reprogramming as a mode of cancer drug resistance. Nature. 2017;546:431-5.

5. Shaked Y. The pro-tumorigenic host response to cancer therapies. Nature reviews Cancer. 2019;19:667-85.

6. Kurppa KJ, Liu Y, To C, Zhang T, Fan M, Vajdi A, et al. Treatment-Induced Tumor Dormancy through YAP-Mediated Transcriptional Reprogramming of the Apoptotic Pathway. Cancer cell. 2020; 37: 104 - 22.e12.

7. Shen CJ, Kuo YL, Chen CC, Chen MJ, Cheng YM. MMP1 expression is activated by Slug and enhances multi-drug resistance (MDR) in breast cancer. PloS one. 2017;12:e0174487.

8. Nieto MA. The snail superfamily of zinc-finger transcription factors. Nature reviews Molecular cell biology. 2002;3:155-66.

9. Li A, Morton JP, Ma Y, Karim SA, Zhou Y, Faller WJ, et al. Fascin is regulated by slug, promotes progression of pancreatic cancer in mice, and is associated with patient outcomes. Gastroenterology. 2014;146:1386-96.e1-17.

10. Chien MH, Lin YW, Wen YC, Yang YC, Hsiao M, Chang JL, et al. Targeting the SPOCK1-snail/slug axismediated epithelial-to-mesenchymal transition by apigenin contributes to repression of prostate cancer metastasis. Journal of experimental clinical cancer research: CR. 2019;38:246.

11. Ye X, Tam WL, Shibue T, Kaygusuz Y, Reinhardt F, Ng Eaton E, et al. Distinct EMT programs control normal mammary stem cells and tumour-initiating cells. Nature. 2015;525:256-60.

12. Chakrabarti R, Hwang J, Andres Blanco M, Wei Y, Lukačišin M, Romano RA, et al. Elf5 inhibits the epithelial-mesenchymal transition in mammary gland development and breast cancer metastasis by 
transcriptionally repressing Snail2. Nat Cell Biol. 2012;14:1212-22.

13. Zhang L, Huang ST, Feng YL, Wan T, Gu HF, Xu J, et al. The Bidirectional Regulation between MYL5 and HIF-1a Promotes Cervical Carcinoma Metastasis. Theranostics. 2017;7:3768-80.

14. Thiery JP. Epithelial-mesenchymal transitions in tumour progression. Nat Rev Cancer. 2002;2:44254.

15. Sternlicht MD, Lochter A, Sympson CJ, Huey B, Rougier JP, Gray JW, et al. The stromal proteinase MMP3/stromelysin-1 promotes mammary carcinogenesis. Cell. 1999;98:137-46.

16. Hung PF, Hong TM, Chang CC, Hung CL, Hsu YL, Chang YL, et al. Hypoxia-induced Slug SUMOylation enhances lung cancer metastasis. 2019;38:5.

17. Lee DG, Lee SH, Kim JS, Park J, Cho YL, Kim KS, et al. Loss of NDRG2 promotes epithelialmesenchymal transition of gallbladder carcinoma cells through MMP-19-mediated Slug expression. Journal of hepatology. 2015;63:1429-39.

18. Shao S, Zhao X, Zhang X, Luo M, Zuo X, Huang S, et al. Notch1 signaling regulates the epithelialmesenchymal transition and invasion of breast cancer in a Slug-dependent manner. 2015; 14: 28.

19. de Azevedo CRAS, Thuler LCS, de Mello MJG, de Oliveira Lima JT, da Fonte ALF, Fontão DFS, et al. Phase II trial of neoadjuvant chemotherapy followed by chemoradiation in locally advanced cervical cancer. 2017; 146: 560-5.

20. Gupta S, Maheshwari A, Parab P, Mahantshetty U, Hawaldar R, Sastri Chopra S, et al. Neoadjuvant Chemotherapy Followed by Radical Surgery Versus Concomitant Chemotherapy and Radiotherapy in Patients With Stage IB2, IIA, or IIB Squamous Cervical Cancer: A Randomized Controlled Trial. 2018; 36: $1548-55$.

21. Chang YS, Jalgaonkar SP, Middleton JD, Hai T. \%J Proceedings of the National Academy of Sciences of the United States of America. Atf3Stress-inducible gene in the noncancer host cells contributes to chemotherapy-exacerbated breast cancer metastasis. 2017; 114: E7159-E68.

22. Volk-Draper L, Hall K, Griggs C, Rajput S, Kohio P, DeNardo D, et al. Paclitaxel therapy promotes breast cancer metastasis in a TLR4-dependent manner. 2014; 74: 5421-34.

23. D'Alterio C, Scala S, Sozzi G, Roz L, Bertolini G. \%J Seminars in cancer biology. Paradoxical effects of chemotherapy on tumor relapse metastasis promotion. 2020;60:351-61.

24. Chiang C, Ayyanathan K. \%J Cytokine, growth factor reviews. Snail/Gfi-1 (SNAG) family zinc finger proteins in transcription regulation, chromatin dynamics, cell signaling, development, and disease. 2013; 24: 123-31.

25. Li CF, Chen JY, Ho YH, Hsu WH, Wu LC, Lan HY, et al. Snail-induced claudin-11 prompts collective migration for tumour progression. 2019; 21: 251-62.

26. Hsu DS, Wang HJ, Tai SK, Chou CH, Hsieh CH, Chiu PH, et al. Acetylation of snail modulates the cytokinome of cancer cells to enhance the recruitment of macrophages. 2014; 26: 534-48.

27. Vandenbroucke RE, Libert C. Is there new hope for therapeutic matrix metalloproteinase inhibition? Nature reviews Drug discovery. 2014;13:904-27. 
28. Castro-Castro A, Marchesin V, Monteiro P, Lodillinsky C, Rossé C, Chavrier P. Cellular and Molecular Mechanisms of MT1-MMP-Dependent Cancer Cell Invasion. Annual review of cell developmental biology. 2016;32:555-76.

29. Huntley GW. Synaptic circuit remodelling by matrix metalloproteinases in health and disease. Nature reviews Neuroscience. 2012;13:743-57.

30. Lerner A, Neidhöfer S, Reuter S, Matthias T. MMP3 is a reliable marker for disease activity, radiological monitoring, disease outcome predictability, and therapeutic response in rheumatoid arthritis. Best practice research Clinical rheumatology. 2018;32:550-62.

31. LaBarge MA. Breaking the canon: indirect regulation of Wnt signaling in mammary stem cells by MMP3. Cell stem cell. 2013;13:259-60.

32. Cheng X, Wei L, Huang X, Zheng J, Shao M, Feng T, et al. Solute Carrier Family 39 Member 6 Gene Promotes Aggressiveness of Esophageal Carcinoma Cells by Increasing Intracellular Levels of Zinc, Activating Phosphatidylinositol 3-Kinase Signaling, and Up-regulating Genes That Regulate Metastasis. Gastroenterology. 2017; 152: 1985-97.e12.

\section{Figures}


A
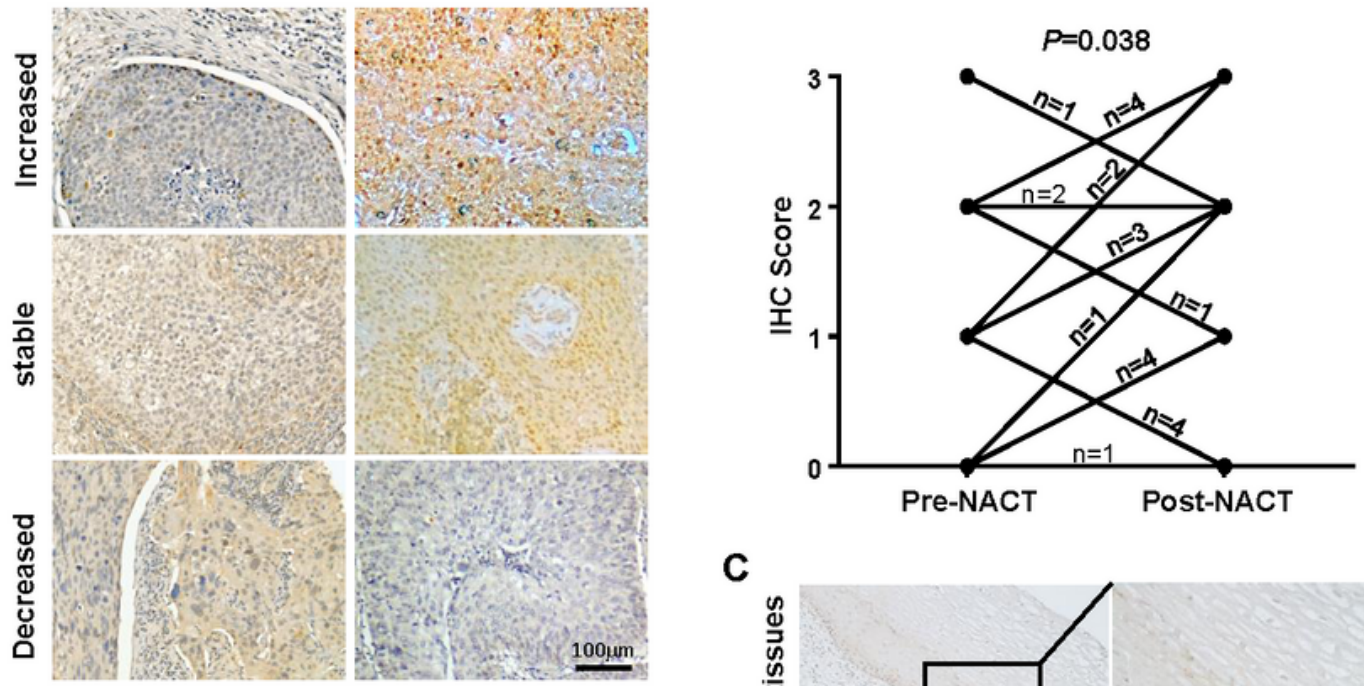

Pre-NACT Post-NACT

B

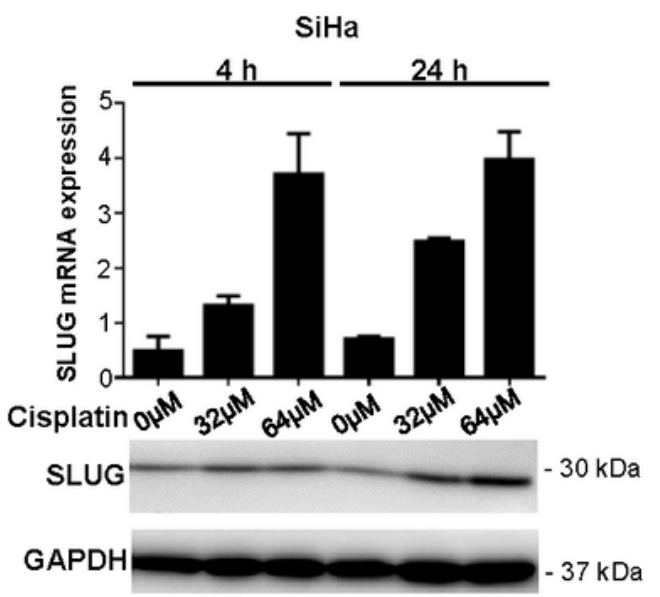

C

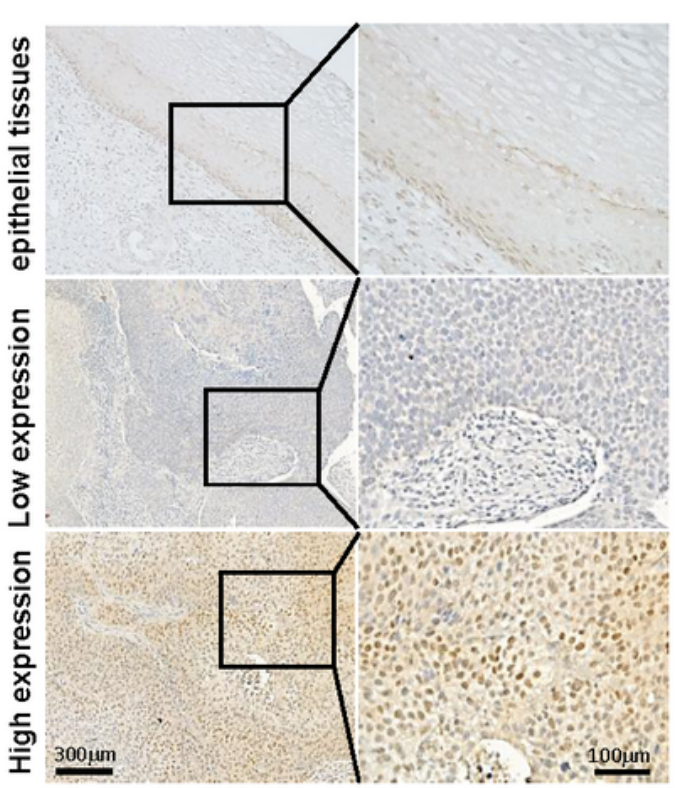

D
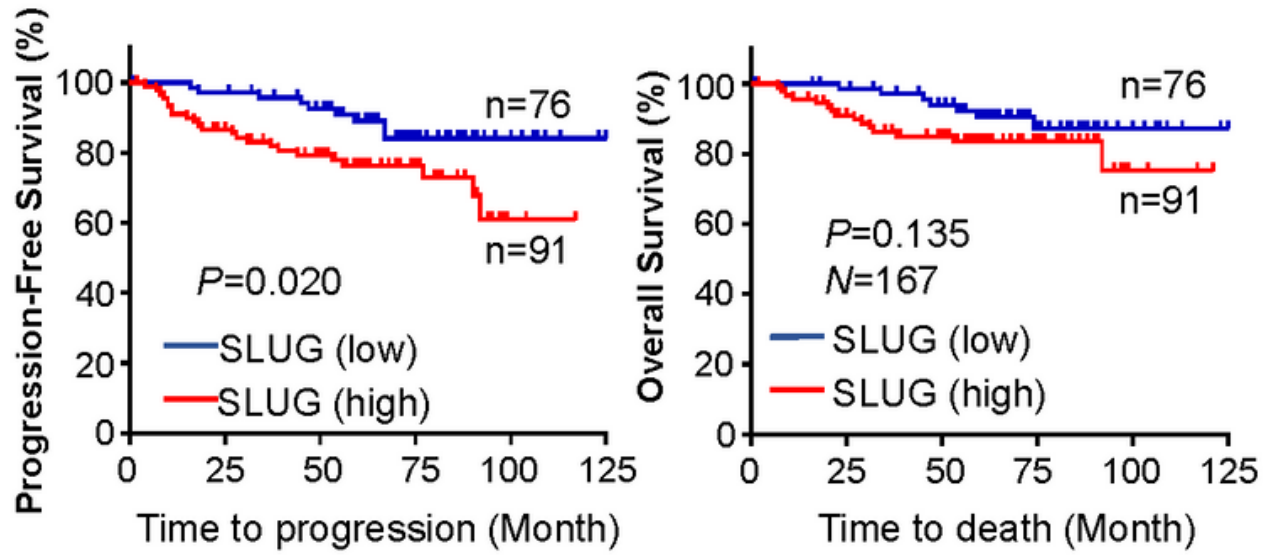

\section{Figure 1}

Chemotherapy enhances SLUG expression in cervical cancer, SLUG expression is associated with poor survival. A. Representative images of SLUG staining in 23 paired pre- and post-NACT cervical cancer tissues. Post-NACT SLUG scores were increased in 14/23 (60.87\%) patients, remained stable in $3 / 23$ $(13.04 \%)$ patients, and decreased in $6 / 23(26.09 \%)$ patients. B. Treated with cisplatin $(0,32,64 \mu \mathrm{M})$ for 4 or 24hours, a dose-dependent increase of SLUG in both mRNA and protein levels was observed in SiHa cells. 
C. Representative images of SLUG staining in adjacent normal epithelial tissues and images of low ,high SLUG staining in the tumor tissues. D. Kaplan-Meier plots of overall survival and disease-free survival of cervical patients stratified by SLUG expression

A

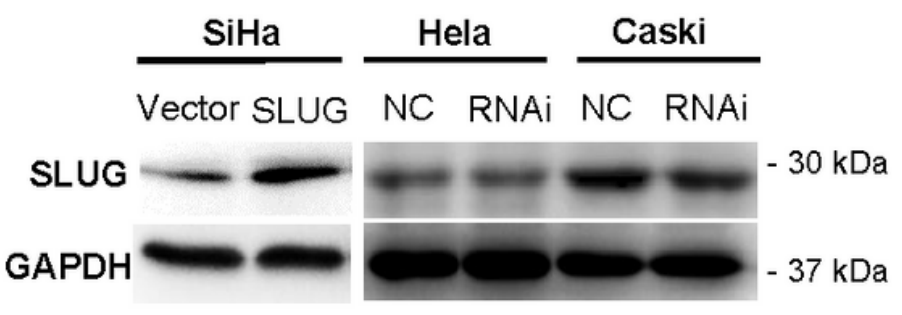

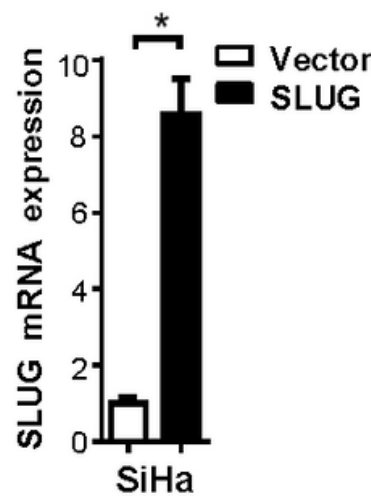

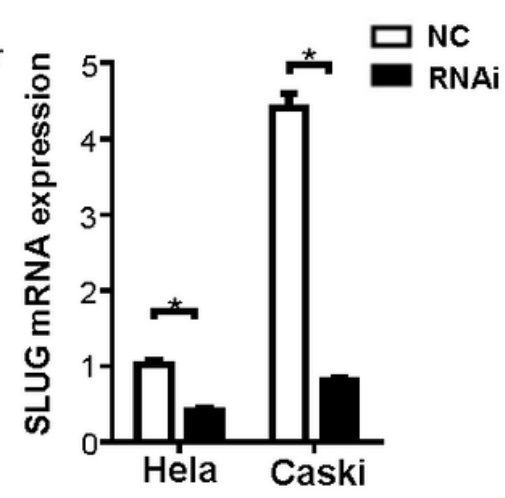

B

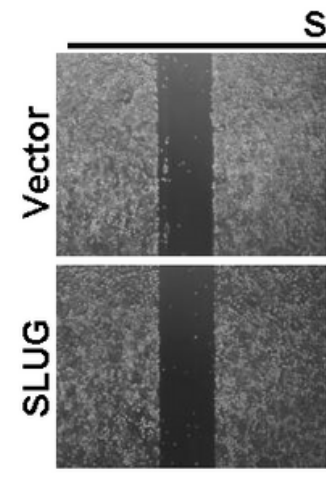

Oh
$\mathrm{SiHa}$

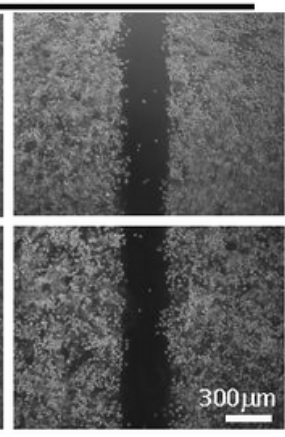

$48 \mathrm{~h}$

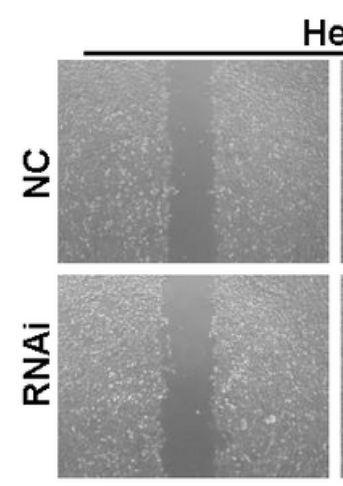

Oh
HeLa

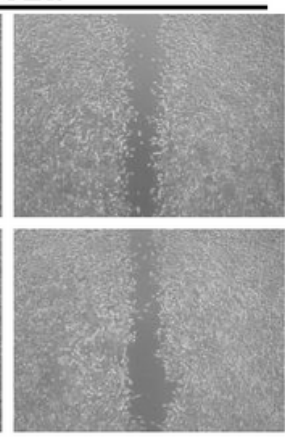

$48 \mathrm{~h}$

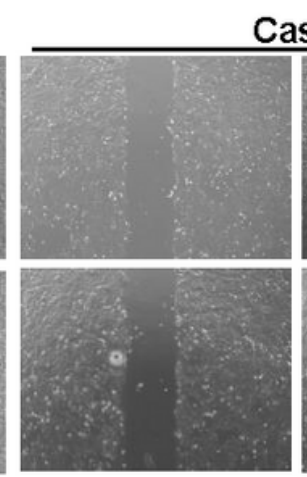

Oh
Caski

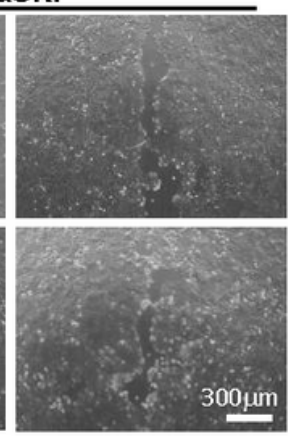

$48 \mathrm{~h}$

C
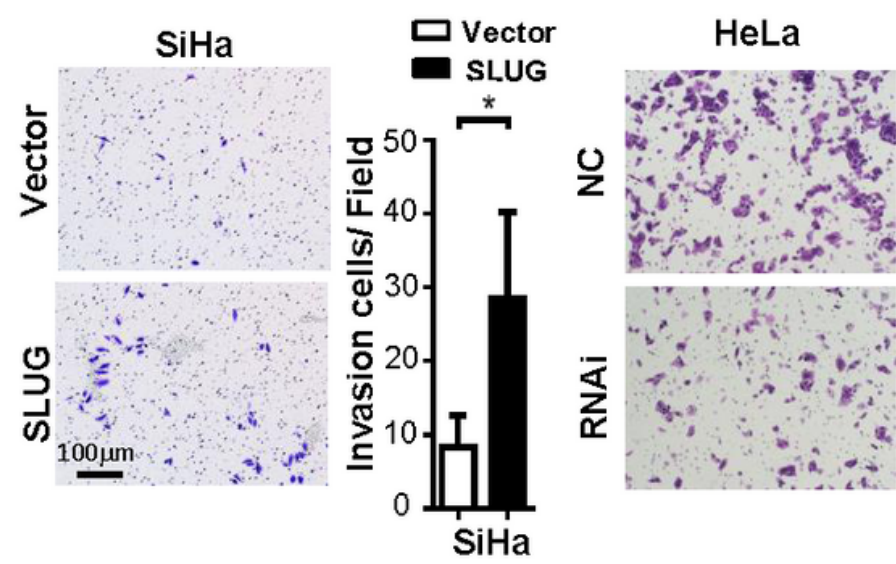

Caski
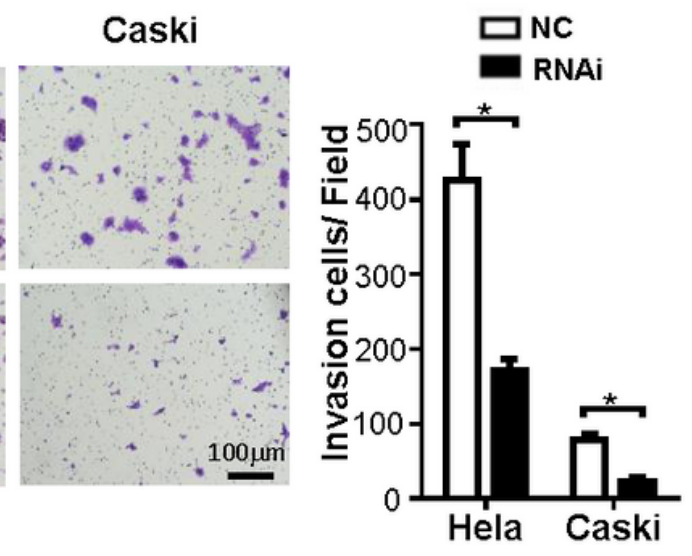

Figure 2

SLUG enhances the invasive capabilities of cervical cancer cells in vitro. A. Western blot and quantitative RT-PCR demonstrate overexpression of SLUG in SiHa cells and silenced SLUG in Caski and HeLa cells. B. Wound healing assays indicated that the cell migratory speed was not changed by SLUG overexpression or knockdown. C. Overexpression of SLUG promotes SiHa cell invasion and silencing of SLUG inhibits HeLa and Caski cells invasion. ${ }^{*} \mathrm{P}<0.05$. 
A

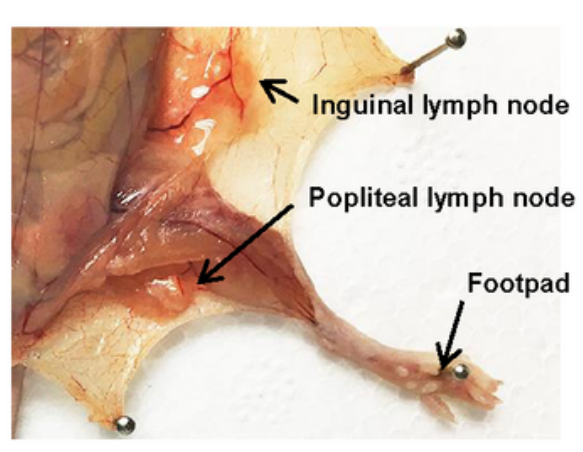

C

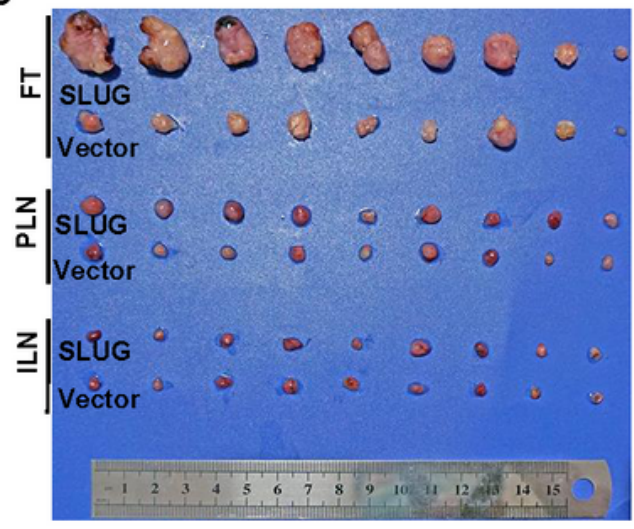

D
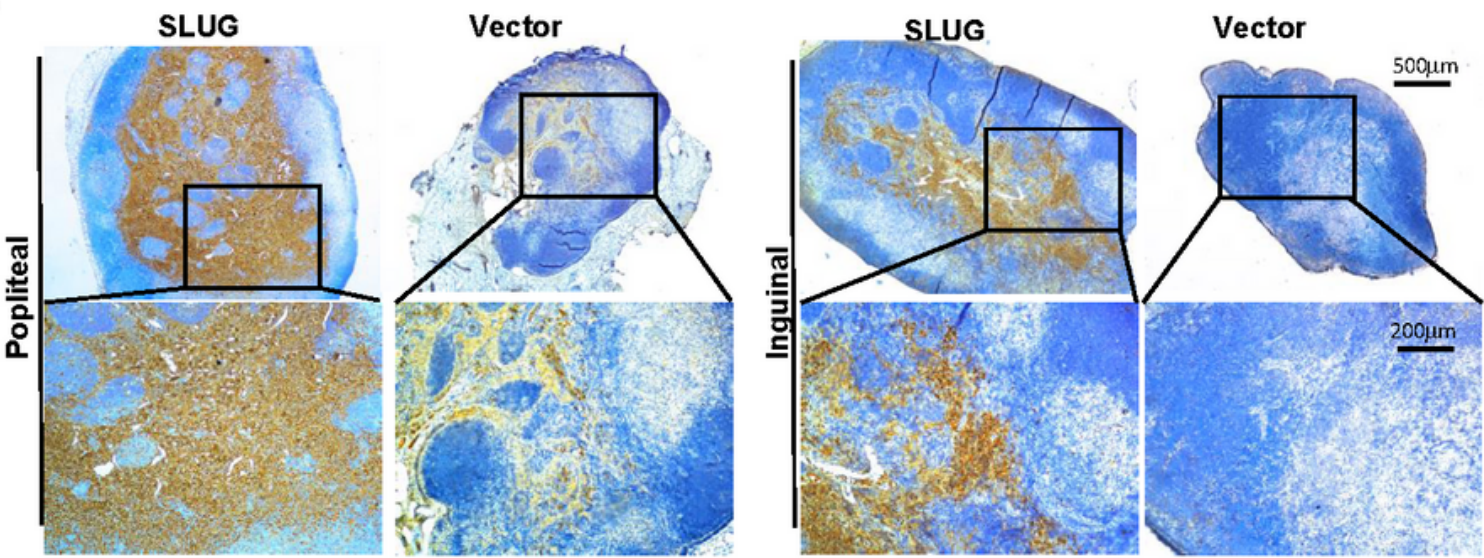

E

Lymph node metastasis in vivo

\begin{tabular}{|c|c|c|c|c|c|c|c|}
\hline \multirow[b]{3}{*}{ Vector } & \multirow{2}{*}{\multicolumn{2}{|c|}{$\frac{\text { No.total LNs }}{\text { Popliteal Inguinal }}$}} & \multirow{2}{*}{\multicolumn{2}{|c|}{$\begin{array}{c}\begin{array}{c}\text { No.metastasis } \\
\text { LNs }\end{array} \\
\text { Popliteal Inguinal }\end{array}$}} & \multicolumn{3}{|c|}{ Metastatic Ratio (\%) } \\
\hline & & & & & Poplite & nguinal & Total \\
\hline & 9 & 9 & 7 & 4 & 77.8 & 44.4 & 61.1 \\
\hline SLUG & 9 & 9 & 9 & 8 & 100.0 & 88.9 & 94.4 \\
\hline
\end{tabular}

\section{Figure 3}

SLUG enhances cervical cancer cells invasive in vivo. A. A lymph node metastasis model was established in BALB/c nude mice and analyzed. B. 8 weeks after 1.5*105 of log-phase SiHa- SLUG(right)/SiHaVector(left) cells were injected into the footpads of nude mice. C. The volumes of footpad tumors, popliteal lymph nodes and inguinal lymph nodes. Right panel shows statistical data. D. Representative images of popliteal/inguinal lymph nodes immunostained with anti-cytokeratin antibody from mice 
inoculated with indicated cells. E. Ratios of metastatic to total dissected popliteal/inguinal lymph nodes from mice inoculated with indicated cells.

A

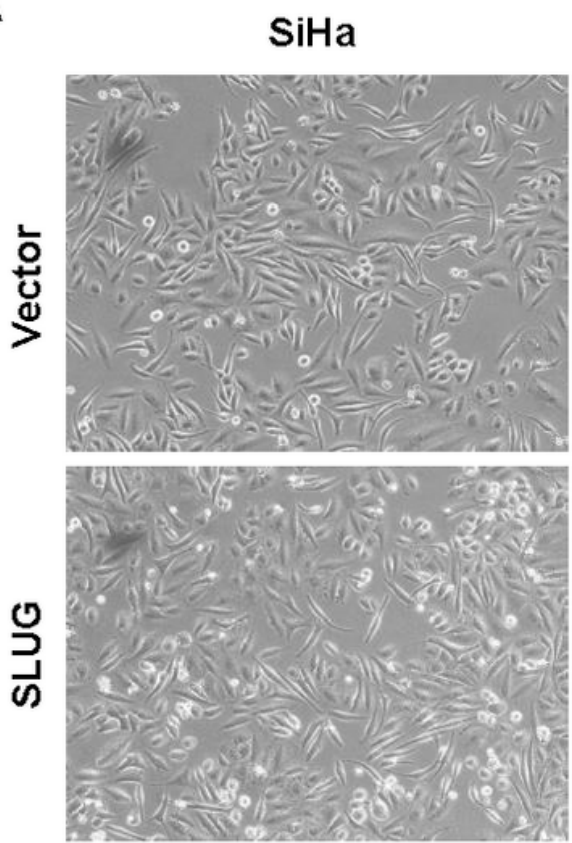

Caski
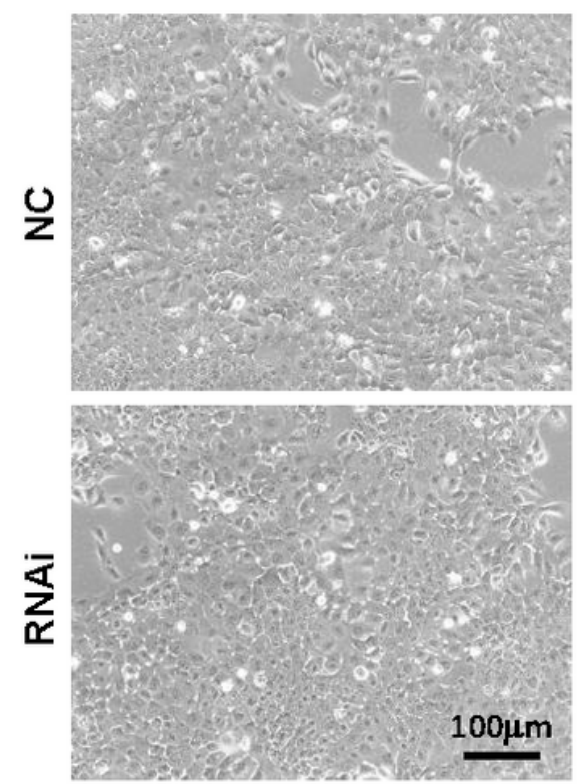

C

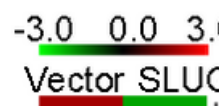

OC100507071

Inc-KLHL25-3

ZSCAN1

LRP1

LEF1-AS1

GNMT

Inc-LIF-1

GK

LOC101929284

Inc-ZG16-2

XLOC I2 009105

LOC4 $4 \overline{0} 0 \overline{867}$

GRAP2

XLOC I2 009216

Inc-ALG14-5

LOC100130373

C20orf173

C20orf173

XLOC I2 013192

PURG

LINC01134

STX18-AS1

Inc-STAB2-1

Inc-PRND-1

STX1B

TEX28

Inc-ZFP42-2

CFHR4

YLPM1

MUC17

Inc-LAMA1-2

ENDOU

XLOC I2_005194

KLHL $\overline{32}$

CAPZB

C6orf10

LOC729696

PTPDC

MMP1

GRAMD1B

PHESOII

AMMP3

DOCK9

SERPINB2

LIPI

PLCB1

Inc-EIF2B5-2

FLJ27255

CCL 3

Inc-PRKD1-8

XLOC I2 002033

Inc-PER2-2

LINC01570

GAB3

\section{GAPDH}

$-37 \mathrm{kDa}$

\section{Figure 4}

SLUG-mediated invasion in cervical cancer cells is independent of EMT. A. No morphological changes in SLUG overexpression SiHa cells and SLUG sikncing Cash cells cells. B. overexpression and knockdown of SLUG failed to alter the EMT maker Vimentin or E-cadherin. C. Heat map showing the most 55 differentially expressed genes in SLUG overexpressing Sib cells and control cells. 
A
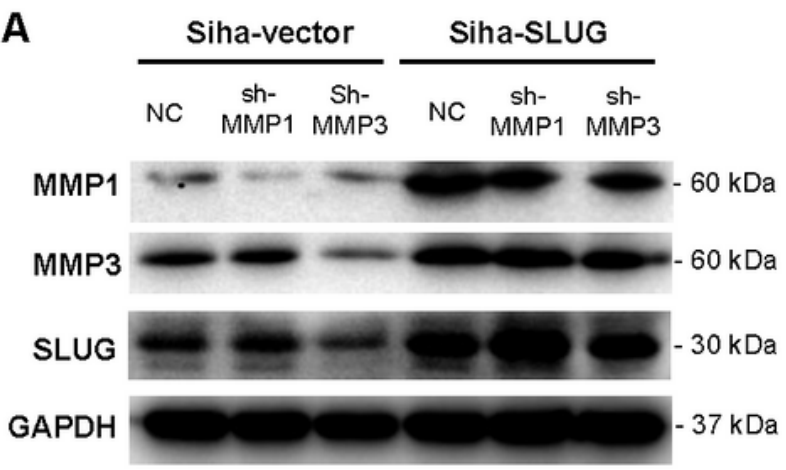

B

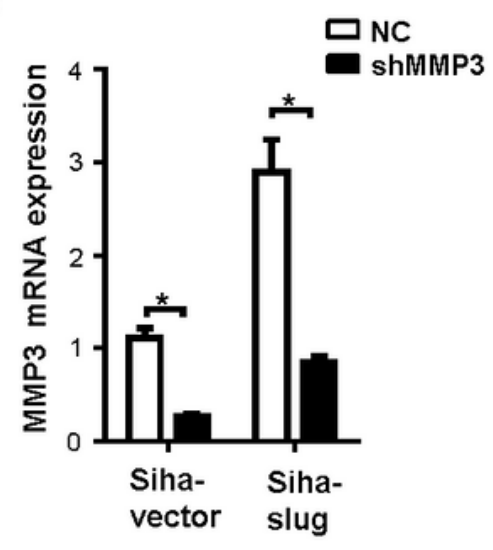

C

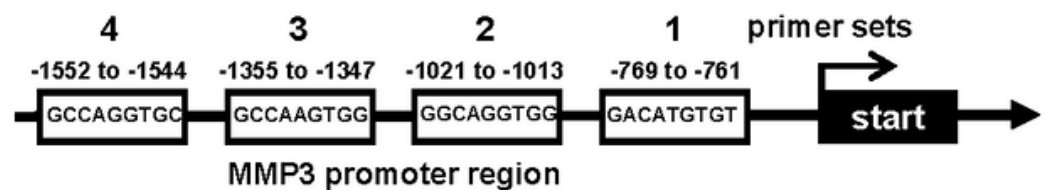

D

\begin{tabular}{|c|c|c|c|c|}
\hline 4 & 3 & 2 & 1 & primer sets \\
\hline-1758 to -1750 & -743 to -735 & -426 to -418 & -408 to -400 & 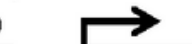 \\
\hline
\end{tabular}

E

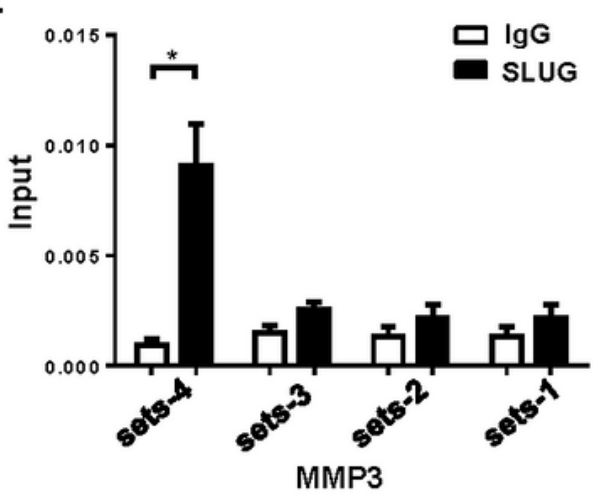

$\mathbf{F}$

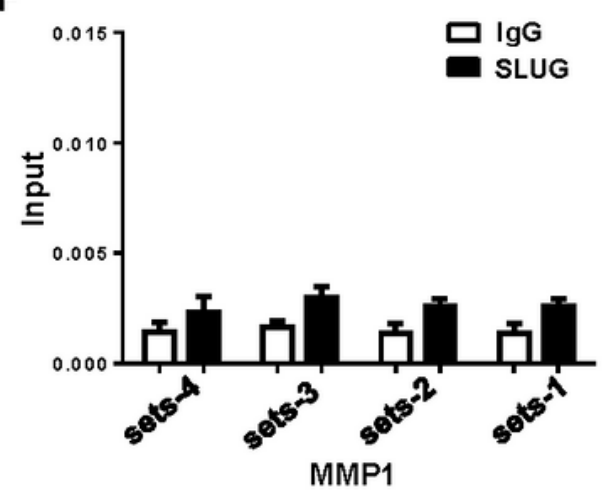

G
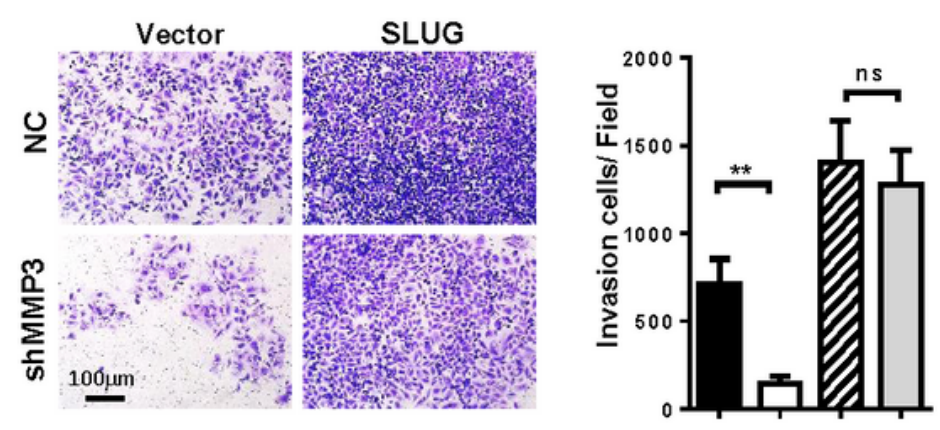

$\square$ Siha-Vector

Siha-Vector-shMMP3

II Siha-SLUG

$\square$ Siha-SLUG-shMMP3

\section{Figure 5}

SLUG upregulates MMP3 in cervical cancer cells. A. Western blot and B. quantitative RT-PCR analysis of MMP3,MMP1 mRNA expression in the indicated cells. C.D.The predicted SLUG binding sites in the promoter region of MMP3/MMP1.Each numbered line above shows the location of a primer pair designed to amplify a region of DNA. E.F. ChIP analysis of SLUG binding on MMP3/MMP1 promoter. ChIP DNA was quantified by real-time PCR primers specific to human MMP3/MMP1 promoter. G. 
Knockdown of MMP3 inhibited SLUG induced cell invasion. Left panel shows representative images of cell invasion. Right panel shows quantified invasion capacities. ${ }^{*} \mathrm{P}<0.05$, ** $\mathrm{P}<0.01$.

A

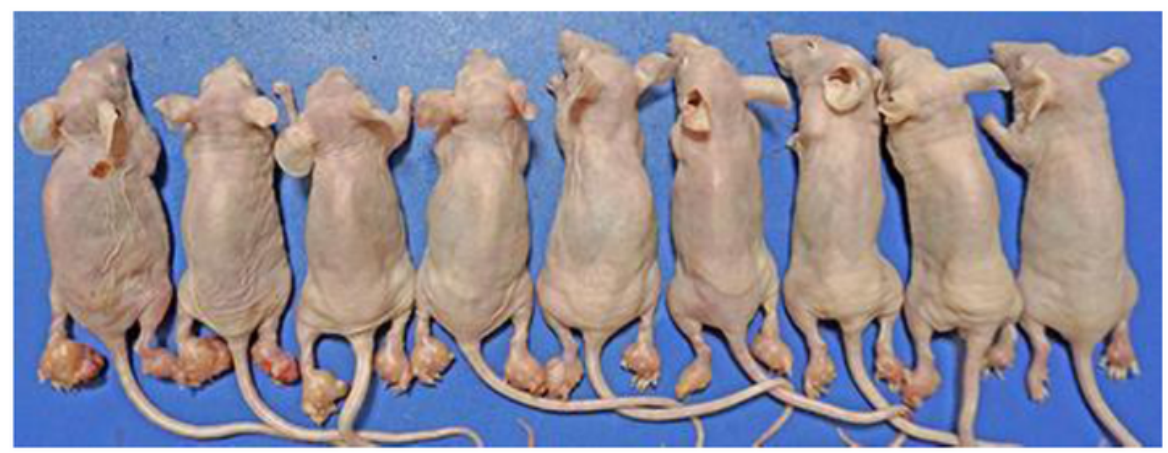

B
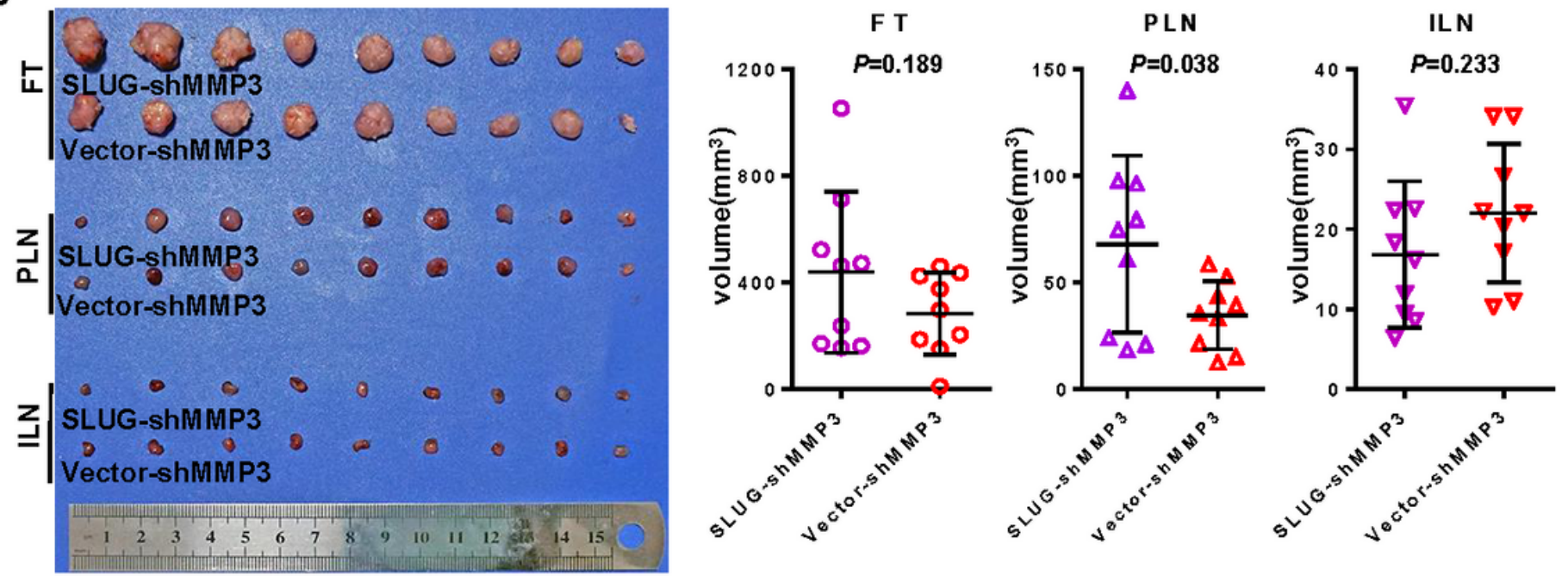

C

Lymph node metastasis in vivo

\begin{tabular}{|c|c|c|c|c|c|c|c|}
\hline \multirow[b]{3}{*}{$\begin{array}{l}\text { Vector- } \\
\text { shMMP3 }\end{array}$} & \multirow{2}{*}{\multicolumn{2}{|c|}{$\frac{\text { No.total LNs }}{\text { Popliteal Inguinal }}$}} & \multirow{2}{*}{\multicolumn{2}{|c|}{$\begin{array}{c}\text { No.metastasis } \\
\text { LNs }\end{array}$}} & \multicolumn{3}{|c|}{ Metastatic Ratio (\%) } \\
\hline & & & & & \multicolumn{2}{|c|}{ Popliteal Inguinal } & \multirow{2}{*}{$\begin{array}{l}\text { Total } \\
50.0\end{array}$} \\
\hline & 9 & 9 & 6 & 2 & 66.7 & 22.2 & \\
\hline $\begin{array}{l}\text { SLUG- } \\
\text { ShMMP3 }\end{array}$ & 9 & 9 & 6 & 3 & 66.7 & 33.3 & 55.6 \\
\hline
\end{tabular}

Figure 6

SLUG upregulates MMP3 in cervical cancer cells. A. 8 weeks after 1.5*105 of log-phase SiHa-SLUGshMMP3(left)/SiHa-Vector-shMMP3(right) were injected into the footpads of nude mice. B. The volumes of footpad tumors, popliteal lymph nodes and inguinal lymph nodes. Right panel shows statistical data.

C. Ratios of metastatic to total dissected popliteal/inguinal lymph nodes from mice inoculated with indicated cells.

\section{Supplementary Files}


This is a list of supplementary files associated with this preprint. Click to download.

- GraphicalAbstract.jpeg

- SupplementaryMaterial.pdf 